\title{
Clinical usefulness of fixation of absorbable implants with cyanoacrylate in comminuted fractures of the maxilla
}

Gang San Ju,

Kyung Min Son,

Woo Young Choi, Ji Seon Cheon

Department of Plastic and Reconstructive Surgery, Chosun University College of Medicine, Gwangju, Korea

\begin{abstract}
Background: The open reduction of craniofacial bone fractures requires internal fixation using metal plates and screws, which have been considered the gold standard. However, metal implants pose a risk of palpation, protrusion, and foreign body reaction, and they may require an additional operation for removal. Recently, good results have been reported for absorbable implants which complement the disadvantages of metal implants. This study presents the results of using absorbable mesh, plates, and screws with cyanoacrylate for more accurate and firmer fixation of comminuted fractures of the maxilla.

Methods: In total, 235 patients underwent operations for comminuted fractures of the maxilla. From January 2012 to December 2014, absorbable mesh and screws were used in 114 patients, while from January 2015 to December 2017, absorbable mesh, plates, and screws with cyanoacrylate were used in 121 patients. Open reduction of the bone fragments was performed, after which absorbable implants were accurately molded and fixed by screws.

Results: All patients underwent postoperative computed tomography scans, which showed highIy accurate reduction and firm fixation in the patients who underwent procedures using absorbable implants, screws, and cyanoacrylate. There were no postoperative complications or cases of abnormal facial contour.

Conclusion: When absorbable implants and screws are used for maxillary fractures, no additional surgery to remove the metal plate is required. In addition, the use of cyanoacrylate enables accurate and firm fixation of the tiny bone fragments that cannot be fixed with screws.
\end{abstract}

Keywords: Absorbable implant / Cyanoacrylate / Fixation / Fractures, comminuted / Maxilla

\section{INTRODUCTION}

The maxilla, which is in the mid-face, has several important functions, including mastication with attached teeth, support-

\footnotetext{
Correspondence: Kyung Min Son

Department of Plastic and Reconstructive Surgery, Chosun University College of Medicine, 365 Pilmun-daero, Dong-gu, Gwangju 61453, Korea

E-mail: 8love17@hanmail.net

This work was supported by research funds from Chosun University Hospital in 2017.

Received June 18, 2019 / Revised August 13, 2019 / Accepted August 18, 2019
}

ing facial structures, and shaping the facial features. However, maxillary fractures can occur from injuries of various causes, potentially requiring surgery.

Maxillary fractures could result in functional changes and facial contour alterations, so ensuring an accurate and solid reduction is important. Using titanium implants for maxillary fracture reduction has been considered the gold standard, with advantages such as a short operating time and solid fixation. However, the disadvantages of titanium implants include the risk of foreign body reaction, palpation of the plates in area 
with thin skin, difficulties in subsequent computed tomography (CT) and magnetic resonance imaging (MRI) scans, the need for secondary surgery to remove them, and hindrance of facial bone growth in children. Recently, studies have investigated the potential of absorbable implants, which do not require secondary surgery for removal (unlike titanium implants), for maxillary fracture reduction [1-4].

Cyanoacrylate is an adhesive used for various tissues, including the skin, bone, and fascia, and its advantages include requiring a shorter time for adhesion than sutures and ease of use [5-8].

During the surgical reduction of comminuted maxillary fractures, larger fragments can be easily reduced and fixed, but it is difficult to reduce small fragments that are not fixed with screws [1-4]. In this study, we report a method of using absorbable implants to avoid secondary surgery for the removal of titanium implants, in combination with cyanoacrylate for accurate and solid reduction and fixation of small bone fragments that are not fixed with screws.

\section{METHODS}

\section{Patients}

The Internal Institutional Review Board of the Korea National Institute of Bioethics Policy (IRB No. 2019-01-014-002) approved this study. Between January 2012 and December 2017, we performed open reduction surgery using absorbable mesh in 235 patients with the following three indications: (1) comminuted fracture of the maxilla; (2) multiple bone fragments of the lateral area of the zygomaticomaxillary buttress; and (3) a lean enough face to palpate the contour of the comminuted fracture. For 114 patients between January 2012 and December 2014, surgery was performed using absorbable mesh and screws. For 121 patients between January 2015 and December
2017, surgery was performed using absorbable mesh, plates, and screws with cyanoacrylate. To ensure accurate diagnoses and evaluations, preoperative CT scans and postoperative follow-up CT scans 5 days after surgery were performed. The patients were followed up for 6 months after surgery. All the operations were performed by a single plastic surgeon (KMS).

\section{Surgical techniques}

Under general anesthesia, the comminuted maxillary fracture site was exposed. Absorbable mesh (poly-lactide-co-glycolide [PLGA] Biobsorb Fixbone plate [Biobsorb Mesh Plating System; Osteonic, Seoul, Korea]) was designed to match the fracture site and cut out with scissors (Fig. 1). After matching the designed mesh to the fracture site, the mesh was soaked in a normal saline bath at $70^{\circ} \mathrm{C}$ and molded against the fracture site. After reduction of the comminuted maxillary fracture, the molded mesh was fixed with absorbable screws.

Between January 2012 and December 2014, after reduction, the absorbable mesh was designed and molded. The broken bone fragments were placed onto the absorbable mesh and fixed with the screws (Figs. 2, 3). The mesh with fixed bone fragments was then placed on the fracture site and fixed with screws.

Between January 2015 and December 2017, cyanoacrylate (LiquiBand, Surgical S [monomeric n-butyl and 2-octyl cyanoacrylates]; Advanced Medical Solutions Ltd., Winsford, UK) was used for accurate and solid reduction and fixation of small bone fragments or thin bone fragments that were not fixed with screws.

After reduction, the absorbable mesh was designed and molded. Small or thin bone fragments were fixed with cyanoacrylate because they could not be fixed with the absorbable mesh and screws. After 30 seconds, forceps were used to check whether these bone fragments were firmly attached. The attached bone
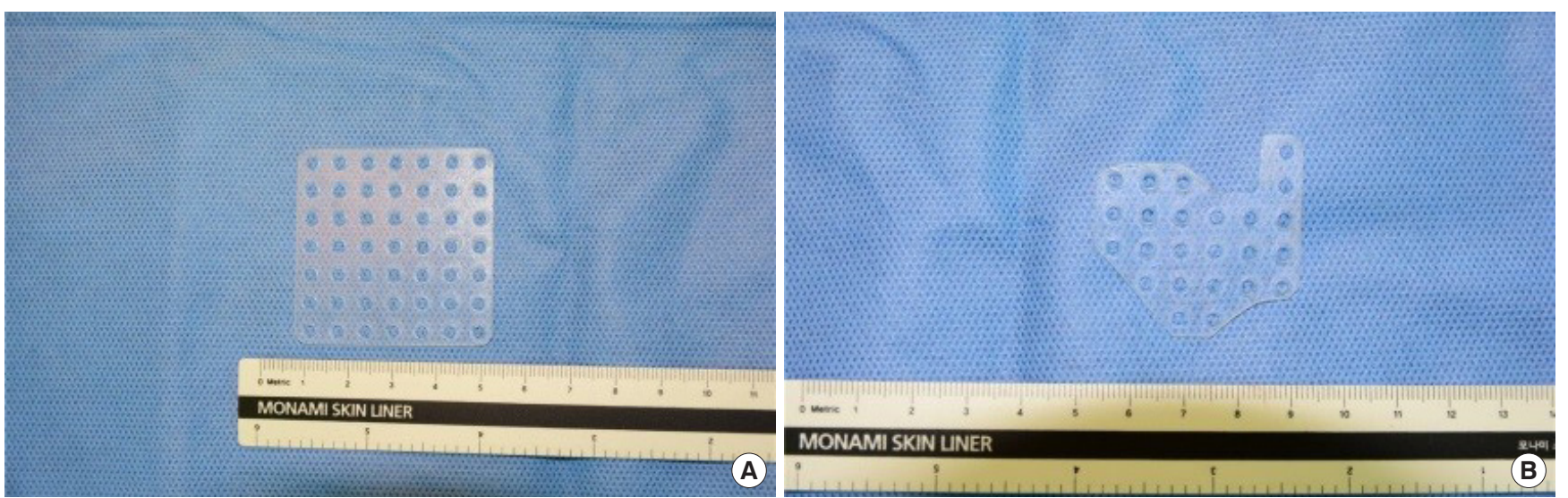

Fig. 1. Shaping of an absorbable mesh plate by cutting with scissors. (A) Before cutting. (B) After cutting. 

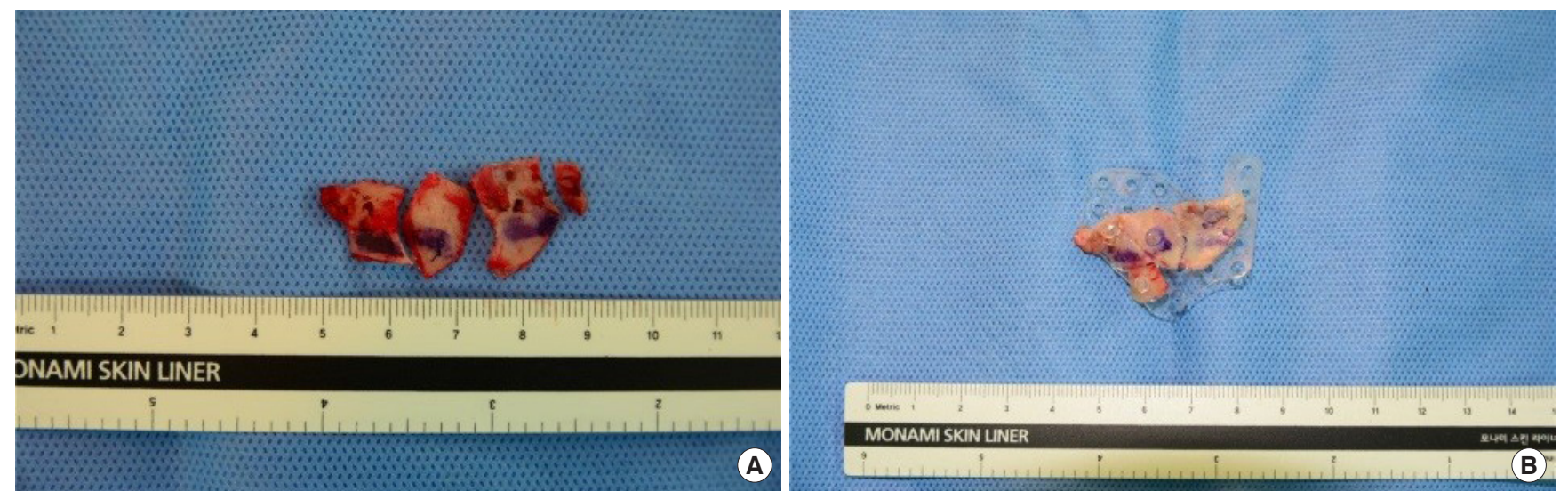

Fig. 2. Fixation of bone fragments. (A) Broken bone fragments of the maxilla. (B) The bone fragments were fixed by an absorbable mesh plate and screws.
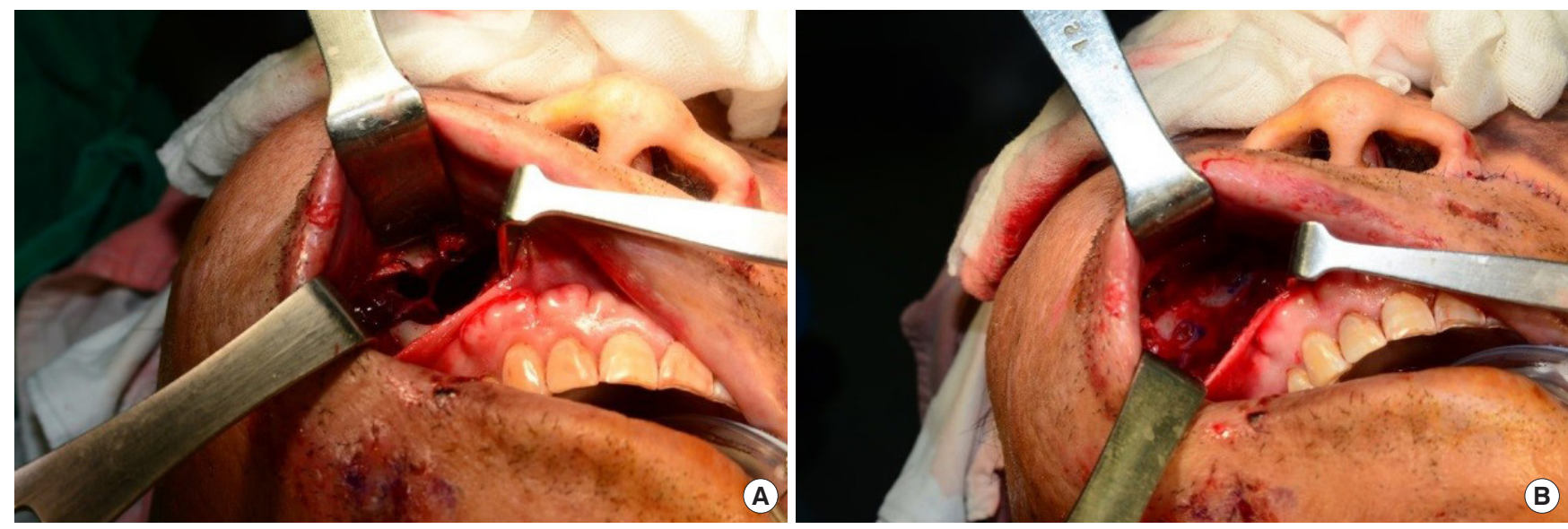

Fig. 3. A patient with a right comminuted fracture of the maxilla. (A) Before reduction. (B) After reduction and fixation by an absorbable mesh without cyanoacrylate.
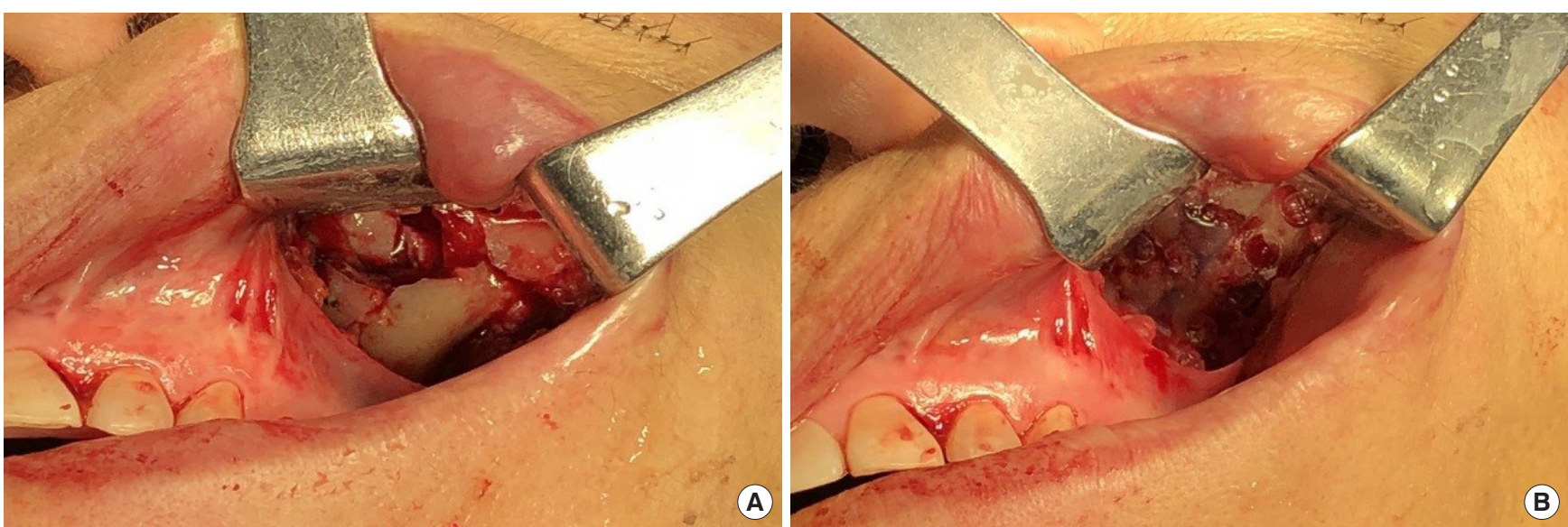

Fig. 4. A patient with a left small comminuted fracture of the maxilla. (A) Before reduction. (B) After reduction and fixation by an absorbable mesh with cyanoacrylate.

fragments were placed onto the absorbable mesh, and fixed with the screws. The mesh with fixed bone fragments was placed on the fracture site and fixed with screws. After absorb- able mesh fixation, cyanoacrylate was applied on top of the bone fragments to reinforce them. The fixation of the fracture site was maintained with cyanoacrylate. In addition, for small 
areas of comminuted fractures, absorbable plates and screws with cyanoacrylate were used for reduction and fixation (Fig. 4).

\section{RESULTS}

In this retrospective cohort study, patients were diagnosed with comminuted maxillary fractures and surgery was performed between January 2012 and December 2017. Between 2012 and 2014, there were 114 patients (98 males and 16 females) who underwent surgery using absorbable mesh and screws. The mean age was 40.2 years for males (range, 13-83 years) and 41.5 years for females (range, 18-74 years). Between 2015 and 2017, there were 121 patients ( 97 males and 24 females) who underwent surgery, with a mean age of 43 years for male patients (range, 14-75 years) and 56.2 years for female patients (range, 20-80 years). Of the 121 patients, 99 underwent surgery using absorbable mesh and screws with cyanoacrylate (79 males and 20 females). The other 22 patients underwent surgery using absorbable plates and screws with cyanoacrylate (18 males and 64 females) (Table 1).

There were no differences between the two groups with regard to any complications, such as malocclusion, infection, enophthalmos, diplopia, numbness and foreign body reaction. Based on the operator's observations with the naked eye, the method using absorbable plates and screws with cyanoacrylate showed

Table 1. Patient demographic by total papulation

\begin{tabular}{|c|c|c|c|}
\hline \multirow{2}{*}{ Material } & \multicolumn{2}{|c|}{ Sex } & \multirow{2}{*}{ Total } \\
\hline & Male & Female & \\
\hline Absorbable mesh plate & 98 & 16 & 114 \\
\hline Absorbable mesh plate+cyanoacrylate & 79 & 20 & 99 \\
\hline Absorbable plate+cyanoacrylate & 18 & 4 & 22 \\
\hline Total & 195 & 40 & 235 \\
\hline
\end{tabular}

more precise alignment. In a comparison between the two groups using preoperative, postoperative, and follow-up CT scans, the use of absorbable plates and screws with cyanoacrylate led to more accurate reduction and solid fixation (Figs. 5, 6).

When only an absorbable plate was used, multiple bone fragments were fixed to the absorbable plate using screws one by one. However, when we used an absorbable plate with cyanoacrylate, we did not have to fix each of the multiple bone fragments using screws one by one. Additionally, cyanoacrylate made it possible for us to fix multiple small bone fragments that could not be fixed with screws. As a result, we saved time and used fewer screws.

\section{DISCUSSION}

Craniofacial fractures are fixed with titanium plates and screws because doing so is relatively quick and leads to rigid fixation. However, titanium plates may be palpable or cause a visible abnormal facial contour, and these plates may hinder CT and MRI scans. When foreign body reactions occur, patients often
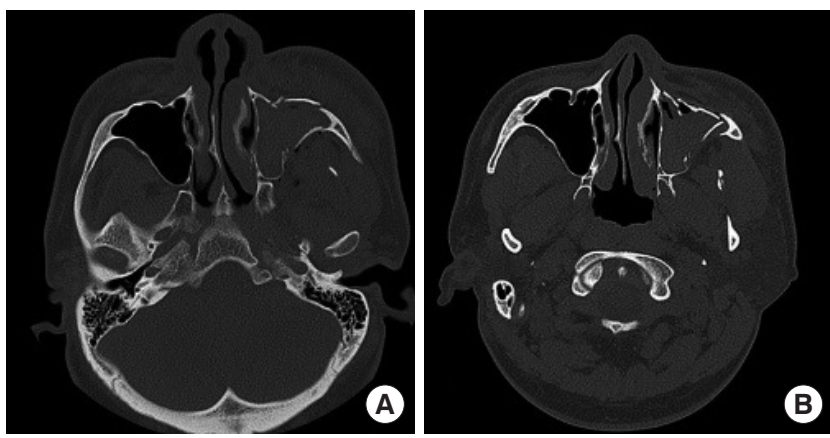

Fig. 5. Computed tomography (CT) scan of a left comminuted fracture of the maxilla treated using an absorbable mesh without cyanoacrylate. (A) Preoperative CT scan. (B) Postoperative CT scan.
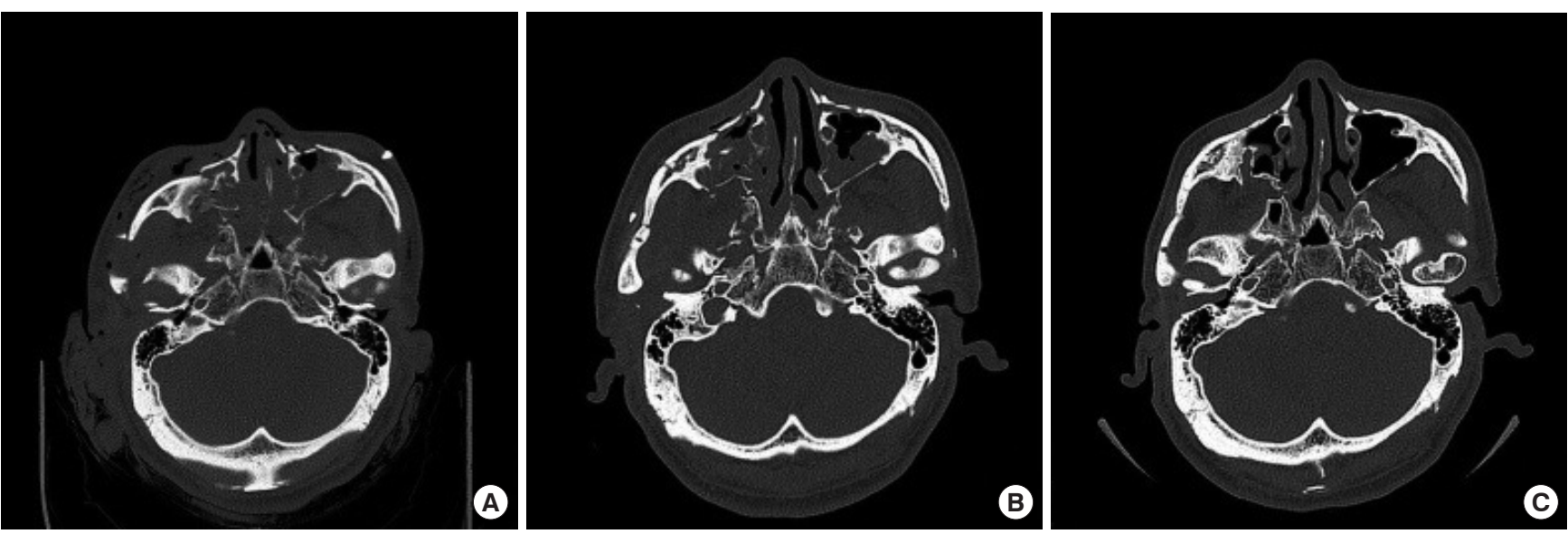

Fig. 6. Computed tomography (CT) scan of a bilateral comminuted fracture of the maxilla using an absorbable mesh with cyanoacrylate. (A) Preoperative CT scan. (B) Postoperative CT scan. (C) Follow-up CT scan 6 months after surgery. 
require secondary surgery to remove titanium plates and screws. Lastly, titanium plates and screws can induce bone deformities in children or adolescents, as their bones continue to grow [3]. To overcome these limitations, some studies have reported the use of absorbable plates and screws for the surgical treatment of craniofacial fractures. These studies have concluded that the use of absorbable plates and screws can produce comparable outcomes to those obtained using titanium plates, while complementing the limitations of titanium plates and screws [3-4,9-11].

Cyanoacrylate has been used as a tissue adhesive for multiple decades, and previous studies have found cyanoacrylate to show satisfactory outcomes, with advantages including a shortened time required for healing, a somewhat lower cost, and aesthetically favorable outcomes [5,12]. In addition to its use for skin sutures, cyanoacrylate is used in various other fields including tympanoplasty, cranial bone flaps, fistula of the meningeal artery, and maxillo-facial complex fractures $[6,8,13]$. Previous studies have described successful procedures yielding rapid and rigid fixation using cyanoacrylate, with no complications.

Although titanium plates and screws are the gold standard for the reduction and fixation of craniofacial fractures, they have some limitations. In a previous study, the authors fixed facial bone fractures using absorbable mesh and screws, which may complement the abovementioned limitations [4].

Another study reported that the thin areas of complex comminuted craniofacial fractures were difficult to reduce. In those cases, titanium plates and screws were utilized to fix larger bones, while small bones were fixed using cyanoacrylate [13].

In our study, we used absorbable mesh, plates, and screws (Biobsorb Fixbone plate) instead of titanium implants for surgery of comminuted maxillary fractures, in order to complement the limitations of titanium implants [4]. After reduction of a comminuted maxillary fracture, cyanoacrylate (LiquiBand, Surgical S [monomeric n-butyl and 2-octyl cyanoacrylates]) was applied to fix fragments of small and thin bones that could be fixed with screws as well as small bone fragments that broke apart during fixation with screws. Previous studies have recommended the use of titanium mesh over absorbable mesh to fix small bone fragments that are not fixed with screws in patients with comminuted maxillary fractures [3,4]. However, by applying the advantageous aspects of cyanoacrylate discussed in the previous study, we successfully reduced and fixed multiple small bone fragments in comminuted maxillary fractures by using absorbable implants with cyanoacrylate [13]. Using absorbable implants with cyanoacrylate might save time and broaden the choice of surgical methods for comminuted maxillary fractures.
As reported in a previous study, although cyanoacrylate is degraded in the body, its exact duration in the body and potential harms have yet to be elucidated [14]. Furthermore, a limitation of this study is that it did not incorporate an objective evaluation of the postoperative facial contour score, fixation strength and bone reduction. To improve these limitations, further studies with objective evaluations would be needed.

Although titanium plates and screws are traditionally used for the fixation of craniofacial fractures, this study suggests that using absorbable implants with cyanoacrylate could overcome the limitations of traditional titanium plates. We used only absorbable mesh instead of titanium plates to overcome these limitations, but multiple small bone fragments or thin bone fragments were difficult to fix with screws. To improve this limitation, we used absorbable implants with cyanoacrylate. As a result, multiple small bone fragments and thin bone fragments could be fixed. Using absorbable implants with cyanoacrylate for fixation of comminuted maxillary fractures might save time and provide an easier, more convenient, and solid surgical method. We hope that this study will provide an additional choice for clinicians who perform facial fracture surgery.

\section{NOTES}

\section{Conflict of interest}

No potential conflict of interest relevant to this article was reported.

\section{Ethical approval}

The study was approved by the Institutional Review Board of the Korea National Institute of Bioethics Policy (IRB No. 201901-014-002) and performed in accordance with the principles of the Declaration of Helsinki. Written informed consents were obtained.

\section{Patient consent}

The patients provided written informed consent for the publication and the use of their images.

\section{ORCID}

Gang San Ju https://orcid.org/0000-0003-1954-7850

Kyung Min Son https://orcid.org/0000-0001-5825-0270

Woo Young Choi https://orcid.org/0000-0001-8849-1569

Ji Seon Cheon https://orcid.org/0000-0001-8555-5088

\section{REFERENCES}

1. O'Connell DA, Futran ND. Reconstruction of the midface and 
maxilla. Curr Opin Otolaryngol Head Neck Surg 2010;18:30410.

2. Hollier LH, Thornton J, Pazmino P, Stal S. The management of orbitozygomatic fractures. Plast Reconstr Surg 2003;111:238692.

3. Kim CH, Kim DH, Kim DH, Song SY. Treatment of frontal sinus fracture using bioabsorbable mesh plates. J Craniofac Surg 2012;23:549-51.

4. Choi WC, Choi HG, Kim JN, Lee MC, Shin DH, Kim SH, et al. The efficacy of bioabsorbable mesh in craniofacial trauma surgery. Arch Craniofac Surg 2016;17:135-9.

5. Grimaldi L, Cuomo R, Brandi C, Botteri G, Nisi G, D’Aniello C. Octyl-2-cyanoacrylate adhesive for skin closure: eight years experience. In Vivo 2015;29:145-8.

6. Gedikli O, Eren SB, Kahya V, Korkut AY, Teker AM, Coskun BU. Efficacy of octyl-2-cyanoacrylate in type I tympanoplasty. J Craniofac Surg 2011;22:1039-41.

7. Tsumoto T, Nakakita K, Hayashi S, Terada T. Bone defect associated with middle meningeal arteriovenous fistula treated by embolization: case report. Neurol Med Chir (Tokyo) 2001;41: 42-7.

8. Sultan A, Mohamed A. Efficacy and safety of using N-butyl cyanoacrylate in cranial fixation following trauma and other pathologies. Turk Neurosurg 2018;28:416-20.
9. Baek WI, Kim HK, Kim WS, Bae TH. Comparison of absorbable mesh plate versus titanium-dynamic mesh plate in reconstruction of blow-out fracture: an analysis of long-term outcomes. Arch Plast Surg 2014;41:355-61.

10. Lee HB, Oh JS, Kim SG, Kim HK, Moon SY, Kim YK, et al. Comparison of titanium and biodegradable miniplates for fixation of mandibular fractures. J Oral Maxillofac Surg 2010;68: 2065-9.

11. Kang IG, Jung JH, Kim ST, Choi JY, Sykes JM. Comparison of titanium and biodegradable plates for treating midfacial fractures. J Oral Maxillofac Surg 2014;72:762.

12. Jan H, Waters N, Haines P, Kent A. LiquiBand ${ }^{\circledR}$ surgical S topical adhesive versus sutures for the closure of laparoscopic wounds: a randomized controlled trial. Gynecol Surg 2013;10: 247-52.

13. Foresta E, Torroni A, Gasparini G, Saponaro G, Longo G, Boniello $\mathrm{R}$, et al. Use of n-butyl-2-cyanoacrylate (Glubran $2\left({ }^{\circledR}\right)$ ) in fractures of orbital-maxillo-zygomatic complex. J Maxillofac Oral Surg 2015;14:761-4.

14. Novotny K, Rocek M, Padr R, Pavlik R, Polovincak M, Adla T, et al. Treating great and small saphenous vein insufficiency with histoacryl in patients with symptomatic varicose veins and increased risk of surgery. Vasa 2018;47:416-24. 